\title{
Geographical Variation in the Use of Diverting Loop Ileostomy in Australia and New Zealand Colorectal Surgeons
}

\author{
David A. Clark ${ }^{1,2,3,4,6}$, Bree Stephensen ${ }^{5}$, Aleksandra Edmundson ${ }^{3,4}$, Daniel Steffens ${ }^{1,2}$, Michael Solomon ${ }^{1,2}$ \\ ${ }^{1}$ Faculty of Medicine and Health, University of Sydney, Sydney; ${ }^{2}$ Surgical Outcomes Research Centre (SOuRCe), Camperdown; ${ }^{3}$ Department \\ of Surgery, Royal Brisbane and Women's Hospital, Brisbane; ${ }^{4}$ St Lucia Campus of University of Queensland, Brisbane; ${ }^{5}$ Department of Surgery, \\ Sunshine Coast University Hospital, Birtinya; ${ }^{6}$ Department of Surgery, St Vincent's Private Hospital Northside, Brisbane, Australia
}

Purpose: Anastomotic leak (AL) after a low pelvic anastomosis is a devastating complication, with short- and long-term morbidity and increased mortality. Surgeons may employ various adjuncts in an attempt to reduce AL rates or mitigate their impact. These include the use of temporary diverting ileostomy (TDI), transanal or rectal tubes and pelvic drains. This questionnaire evaluates the preferences and routine use of these adjuncts in Australasian colorectal surgeons.

Methods: A cross-sectional survey was administered to Australian and New Zealand colorectal surgeons on September 20 , 2018. The study survey consisted of 15 questions exploring basic demographics and the number of rectal resections and ileal pouches performed in 12 months, along with the surgeon's preference for the use of diverting stomas, rectal tubes, and pelvic drains.

Results: There were 90 respondents to the survey (31.6\%). Surgeons in Western Australia (71.4\%) were more likely to use a mandatory TDI in colorectal extraperitoneal anastomoses than surgeons in Queensland (14.3\%). South Australian surgeons are more likely to employ a mandatory TDI (100\%) for ileal pouches than Queensland surgeons (42.9\%). Rectal tubes are not commonly utilized (40.0\% never use them), and pelvic drains are ( $45.6 \%$ in all cases). Surgeons consider a median AL rate of $15 \%$ was felt to justify the use of a TDI in low pelvic anastomoses and a median AL rate of $10 \%$ for ileal pouches

Conclusion: There is considerable geographical variation in colorectal surgical practice throughout Australia and New Zealand. While surgeons interrogate the same literature, there are presumably other factors that see translation into variations in clinical practice.

Keywords: Ileostomy; Trans anal tube; Anastomotic leak; Pelvic drain; Restorative proctocolectomy

\section{INTRODUCTION}

Anastomotic leak (AL) after a low pelvic anastomosis is a devastating complication, with short- and long-term morbidity and in-

Received: Jun 12, 2020 - Revised: Sep 9, 2020 - Accepted: Sep 14, 2020 Correspondence to: David A. Clark, MBBS, FRACS, FRCS, Ed Department of Surgery, St Vincent's Private Hospital Northside, 627 Rode Road, Chermside, 4032 Brisbane, Queensland, Australia

Tel: +61-7-3350-2088, Fax: +61-7-3350-2333

E-mail: David.Clark@doctors.org.uk

ORCID: https://orcid.org/0000-0002-2065-3012

(C) 2021 The Korean Society of Coloproctology

This is an open-access article distributed under the terms of the Creative Commons Attribution NonCommercial License (https://creativecommons.org/licenses/by-nc/4.0) which permits unrestricted noncommercial use, distribution, and reproduction in any medium, provided the original work is properly cited. creased mortality. Following low anterior resection for rectal cancer, reported AL rates average $11 \%$ to $15 \%$ [1]; but when asymptomatic AL are included, reported rates are higher [2].

Ileal pouch anal anastomoses (IPAA) performed after restorative proctocolectomy has seen varying leak rates reported. The Lovegood meta-analysis, which included 4,183 patients, reported an AL rate of $6.9 \%$ with a pelvis sepsis rate of $7.2 \%$ [3]. Significant functional sequelae have been observed and up to $40 \%$ of these patients come to reoperation and eventually pouch excision [4].

Surgeons may employ various adjuncts in an attempt to reduce AL rates or mitigate their impact. These include the use of temporary diverting ileostomy (TDI), pelvic drains, and transanal or rectal tubes [5].

TDI has been widely used to protect a low pelvic anastomosis. 
While it is considered that a TDI does not prevent AL, there is reasonable evidence that it reduces the clinical impact of the leak $[6,7]$. However, analysis of the Dutch TME trial showed a $19 \%$ permanent stoma rate [8]. Readmission rates are high, with a recent large retrospective review showing a $28 \%$ readmission rate within 60 days, most commonly for dehydration [9] or obstruction [10]. Fielding et al. [11] reported a 2.2-fold increase in rates of new or worsening chronic kidney disease compared with patients without an ileostomy and that these findings persist despite the closure of the ileostomy.

Similarly, studies in ileal pouch surgery have shown that a TDI does not reduce the likelihood of AL [12]. Diverting ileostomies for ileal pouch surgery are even more problematic, given their proximal location and consequent issues with high output. Closure of the ileostomy requires a second procedure with associated morbidity and mortality. Furthermore, Sugerman et al. [13] noted that it is technically difficult to bring out an ileostomy in an obese patient with a large amount of subcutaneous tissue. With the ileal pouch bow-stringing the ileal mesentery to the retroperitoneum, tension is created when securing the ileostomy to the skin level and the stoma is often recessed.

Pelvic drain use remains controversial. A 2017 review assessed 7 systematic reviews, 1 randomized controlled trial and 7 cohort studies, concluded that that routine drainage has no significant impact on the rate of colorectal AL but may have a selective utility when the operative field is not dry [14]. The included GRECCAR 5 randomized controlled trial of pelvic drains reported no difference in the drained or undrained groups [15]. Earlier reviews have reported a lower AL rate and lower intervention rate in drained patients [16].

In a randomized controlled study, Xiao et al. [5] showed that rectal tubes were safe and decreased the incidence of clinically significant AL (4\% in the rectal tube group vs. $9.6 \%$ in the control group, $\mathrm{P}=0.026$ ). A 2016 meta-analysis, including 909 patients, supported these findings and reported both a reduction in $\mathrm{AL}$ rate and the need for reoperation [17].

The use of both transanal tubes and pelvic drains among Australian and New Zealand colorectal surgeons has not previously been investigated, and it is unclear what effect recent negative studies on pelvic drains may have had on local practice. We devised a questionnaire to administer to members of the Colorectal Surgical Society of Australia and New Zealand (CSSANZ), evaluating preferences and routine use of these adjuncts in their usual practice.

\section{METHODS}

\section{Study design}

A cross-sectional survey was administered among Australian and New Zealand colorectal surgeons in 2019. St Vincent's Private Hospital Northside Human Research and Ethics committee approved the study protocol (No. HREC 18/21). This was a volun- tary study. Consent was confirmed at invitation and participation in the survey confirmed consent. This was outlined in the requirement of participation.

\section{Participants}

Surgeons, consultants, and fellows $(\mathrm{n}=285)$ registered with CSSANZ were invited by e-mail to participate in this study online requiring the completion of a short survey taking approximately 3 to 4 minutes to complete. All invited surgeons were assigned a unique 3-digit code known to a third party (research administration of CSSANZ) administering the survey on behalf of the investigators. The invitation outlined the purpose of the survey and the requirements for participation.

\section{Study survey}

The study survey consisted of 2 main sections. There were 15 questions. The first section captured basic demographics, including surgeons' age, time as consultant, and location of the practice. The second section captured clinical information, including the number of rectal resections and ileal pouches performed in 12 months, along with the surgeon's preference for the use of rectal tubes, diverting stomas, and drains (Appendix 1). A reminder was sent by e-mail 2 weeks after the initial invitation. Preliminary testing of the survey was conducted among 13 colorectal surgeons who completed the pilot survey while attending a local professional meeting in Brisbane. Clarity, feasibility, and feedback were assessed, showing acceptability and willingness to participate.

The CSSANZ Research Support Committee assisted with the distribution of the study survey to CSSANZ members by directly emailing surveys to colorectal fellows and practicing colorectal surgeons. The investigators did not have access to the members' personal details. All members of CSSANZ were deidentified by a third party by the assignment of a unique study code, unknown to the investigators. Surveys were designed using Survey Monkey (Palo Alto, CA, USA) and electronically distributed with the assistance of the CSSANZ Research Support Committee.

\section{Statistical methods}

Descriptive statistics were computed for all measurements and displayed graphically. GraphPad Prism ver. 8.0 (GraphPad, San Diego, CA, USA) and Stata software packages (StataCorp LLC, College Station, TX, USA) were used for analysis. A P-value of $<0.05$ was considered significant. A 2-side Fisher exact test was used for analysis. The analysis was performed on the respondents overall and raw figures presented for the subset of high-volume surgeons defined as performing $>25$ rectal resections per year.

\section{RESULTS}

\section{Characteristics of the study sample}

The study survey was sent by e-mail to 285 members of the CSSANZ, with 90 respondents (31.6\%) completing the survey. All 
Table 1. Characteristics of responding surgeons

\begin{tabular}{lcccc}
\hline Characteristic & $\begin{array}{r}\text { Overall } \\
(\mathrm{n}=90)\end{array}$ & $\begin{array}{c}\text { Colorectal } \\
\text { fellows } \\
(\mathrm{n}=11)\end{array}$ & $\begin{array}{c}\text { Consultants } \\
(\mathrm{n}=79)\end{array}$ & P-value \\
\hline Age (yr) & $48(41-54)$ & $36(35-38)$ & $49(44-56)$ & $<0.001$ \\
Consultant practice (yr) & - & - & $14(8-20)$ & - \\
$\begin{array}{l}\text { Number of rectal resections/yr } \\
\text { Number of ileal pouches/yr }\end{array}$ & $20(15-30)$ & $20(12-30)$ & $20(15-30)$ & 0.846 \\
$\begin{array}{l}\text { lleal pouches/yr } \\
\geq 6\end{array}$ & $8(0.9)$ & $0(0)$ & $2(0-4)$ & 0.990 \\
$<6$ & $82(91.1)$ & $11(100)$ & $71(89.9)$ & \\
\hline
\end{tabular}

Values are presented as median (interquartile range) or number (\%).

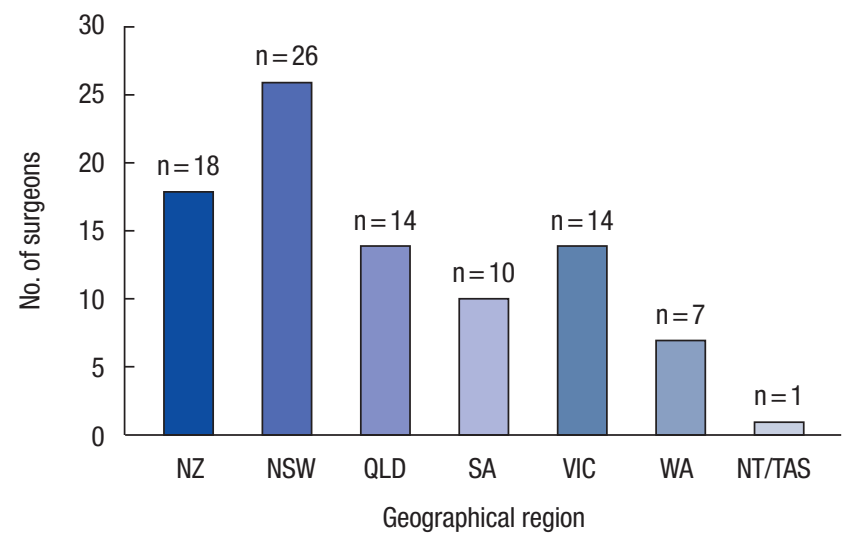

Fig. 1. Geographical distribution of participating surgeons. NZ, New Zealand; NSW, New South Wales; QLD, Queensland; SA, South Australia; VIC, Victoria; WA, Western Australia; NT/TAS, Northern Territory/Tasmania.

respondents answered all questions. A demographic summary of participating surgeons is illustrated in Table 1. The geographical distribution of the respondents in this study is displayed in Fig. 1.

\section{Use of a temporary diverting ileostomy for low pelvic anastomoses}

Surgeons in Western Australia (5 of 7, 71.4\%) were more likely to use a mandatory TDI in low and ultra-low anterior resections than surgeons in Queensland (2 of 14, 14.3\%). Overall, 37.8\% of surgeons employed a mandatory TDI with all low or ultra-low anterior resections. While there is geographical variation in practice, this overall variation does not reach statistical significance ( $\mathrm{P}=0.056)$ (Fig. 2).

When high-volume surgeons were considered (annual caseload $\geq 25$ low and ultra-low anterior resections; $\mathrm{n}=38$ ), it was observed that overall $32.0 \%$ of these surgeons employed a mandatory TDI. Similarly, $42.0 \%$ of surgeons $(n=52)$ with a lower annual caseload $(<25$ rectal resections/year) employed mandatory TDI with low

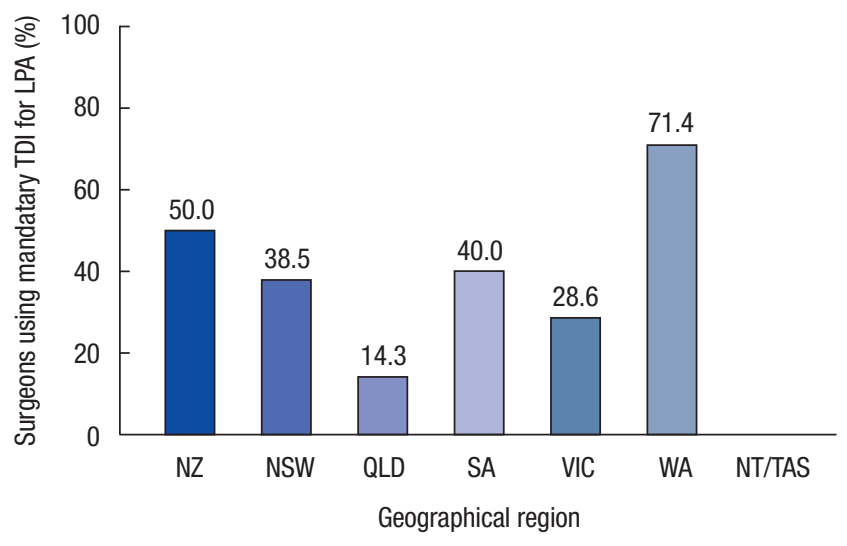

Fig. 2. Percentage of surgeons using mandatory temporary diverting ileostomy (TDI) for low or ultra-low pelvic anastomoses. LPA, low pelvic anastomoses; NZ, New Zealand; NSW, New South Wales; QLD, Queensland; SA, South Australia; VIC, Victoria; WA, Western Australia; NT/TAS, Northern Territory/Tasmania.

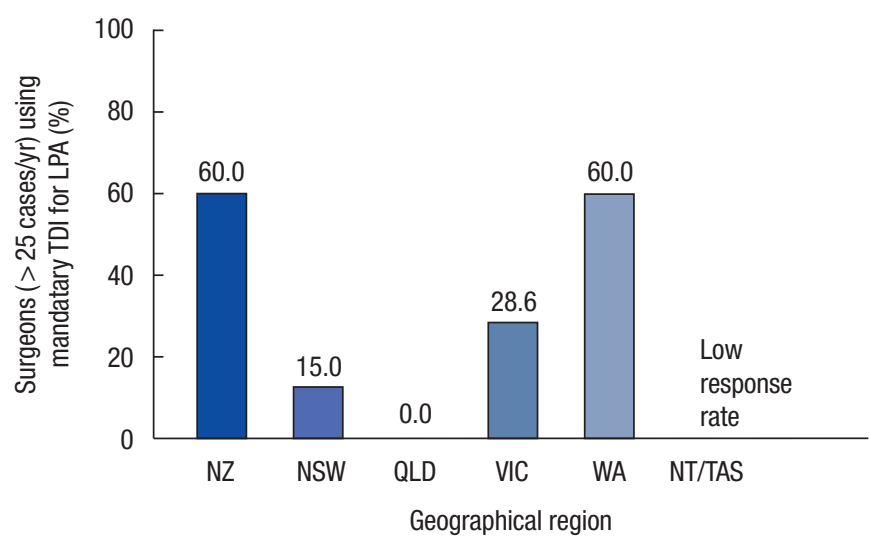

Fig. 3. Percentage of high-volume surgeons using mandatory temporary diverting ileostomy (TDI) for low or ultra-low anterior resections (with a high caseload $>25$ /year). LPA, low pelvic anastomoses; NZ, New Zealand; NSW, New South Wales; QLD, Queensland; VIC, Victoria; WA, Western Australia; NT/TAS, Northern Territory/Tasmania.

and ultra-low resections $(\mathrm{P}=0.172)$. None of the high-volume Queensland colorectal surgeons employed a mandatory TDI (Fig. 3).

\section{Use of a temporary diverting ileostomy for ileal pouch surgery}

Surgeons appear to be more conservative when it comes to ileal pouch surgery. The geographical influence on the use of TDI was also observed for ileal pouch surgery $(\mathrm{P}=0.029)$, with surgeons in Queensland preferring a more selective approach to its use (Fig. 4). Due to the low number of respondents from NT (1) and Tasmania (0), the responses from these regions were not considered in this analysis. 


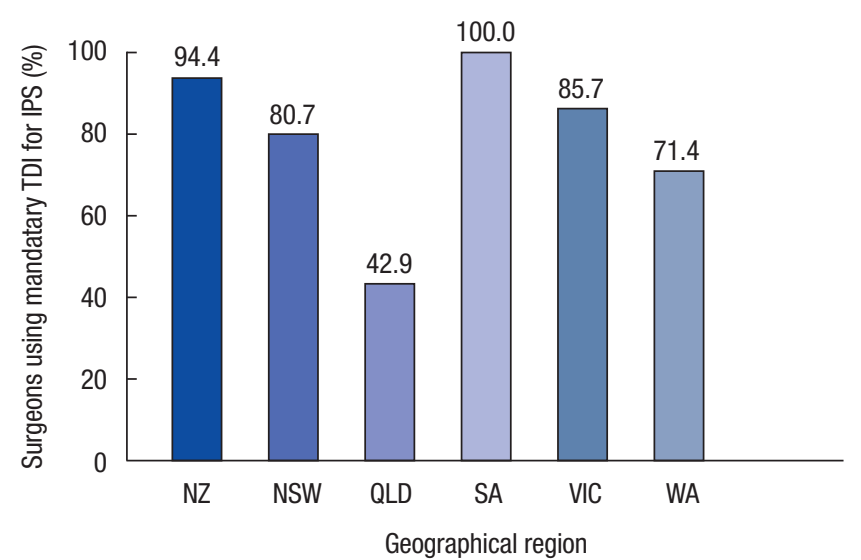

Fig. 4. Percentage of surgeons using a mandatory temporary diverting ileostomy (TDI) for ileal pouch surgery (IPS). NZ, New Zealand; NSW, New South Wales; QLD, Queensland; SA, South Australia; VIC, Victoria; WA, Western Australia.

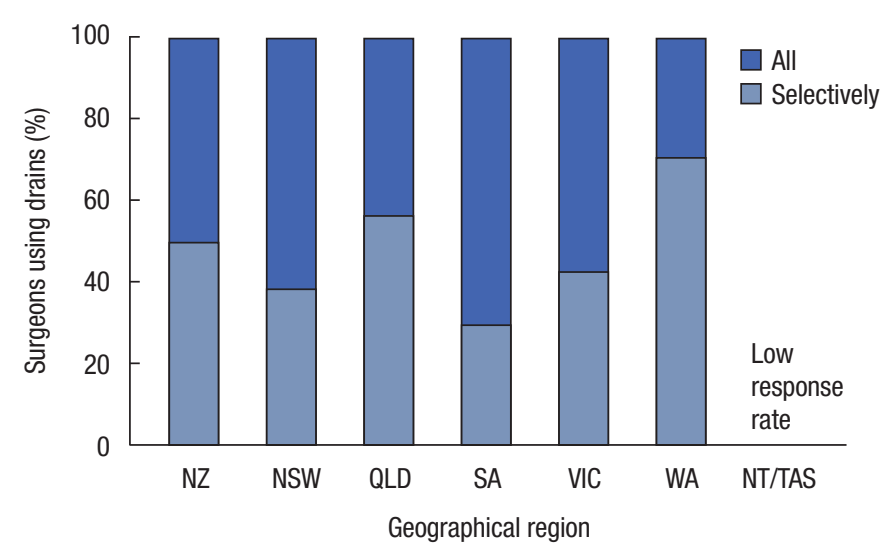

Fig. 5. Regular use of pelvic drains by colorectal surgeons. NZ, New Zealand; NSW, New South Wales; QLD, Queensland; SA, South Australia; VIC, Victoria; WA, Western Australia; NT/TAS, Northern Territory/Tasmania.

\section{Use of pelvic drains}

The majority of surveyed surgeons still use pelvic drains either routinely or selectively. No respondents had a policy of never using a drain (Fig. 5). Overall, $45.6 \%$ of surgeons use a pelvic drain for all cases, and the remaining $54.4 \%$ report using pelvic drains selectively.

\section{Use of transanal rectal tubes}

The use of rectal tubes in colorectal surgery may be influenced by geographical location. More surgeons practicing in Queensland choose to use rectal tubes in low pelvic anastomoses and ileal pouch operations than other surveyed regions of Australia and New Zealand (Table 2). Surgeons choosing to use rectal tubes reported they usually leave them in place for 5 days. Forty percent of surveyed surgeons never use rectal tubes.
Table 2. The geographical distribution of surgeons' use of rectal tubes in low pelvic anastomoses and ileal pouch operations

\begin{tabular}{lcc}
\hline \multirow{2}{*}{ Geographical region } & \multicolumn{2}{c}{ Surgeons using rectal tubes (\%) } \\
\cline { 2 - 3 } & All low pelvic anastomoses & All ileal pouch operations \\
\hline New Zealand & 33.3 & 55.6 \\
New South Wales & 3.8 & 19.2 \\
Queensland & 78.6 & 78.6 \\
South Australia & 0.0 & 40.0 \\
Victoria & 0.0 & 21.4 \\
Western Australia & 14.3 & 14.3 \\
\hline
\end{tabular}
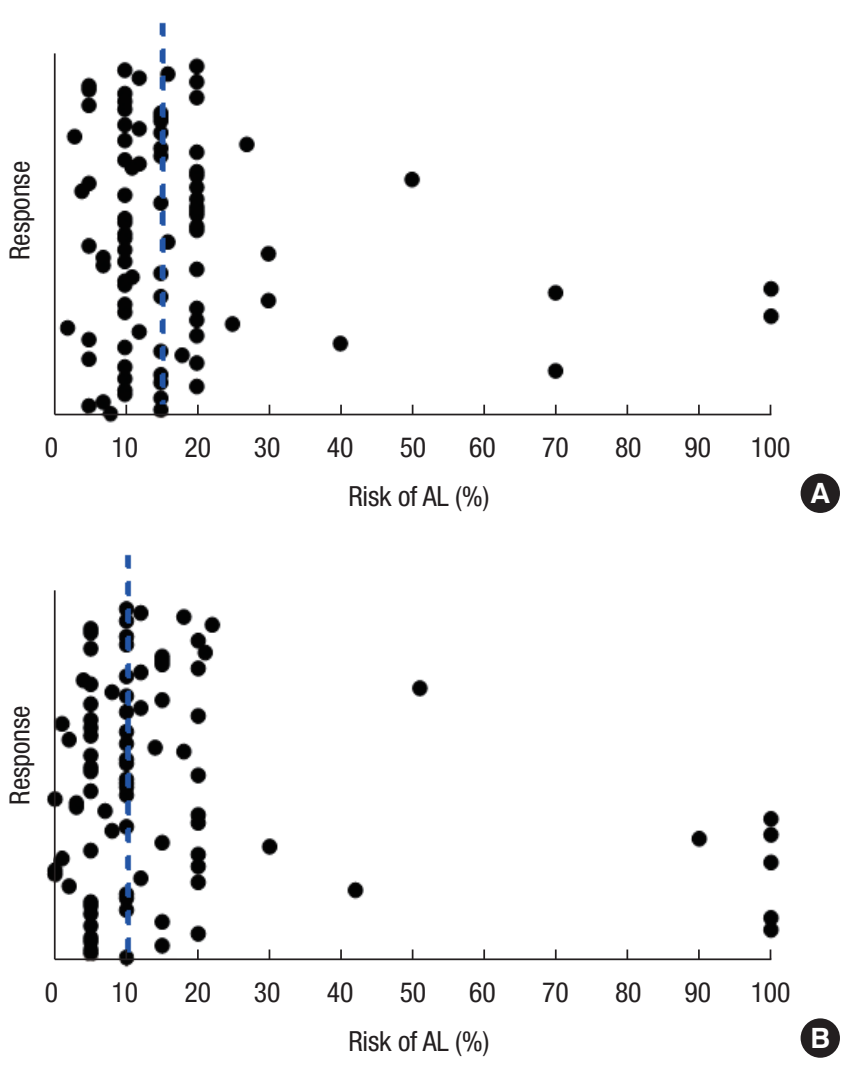

Fig. 6. Distribution of respondents. Surgeons were asked at what percentage risk, on a visual analogue scale, of anastomotic leak (AL) they would consider a diverting ileostomy to be in their patient's best interest. (A) Low pelvic colorectal anastomosis. (B) Ileal pouch surgery. Blue dotted line marks median risk.

\section{Risk of anastomotic leak and diverting ileostomy}

The assessment of risk of anastomotic dehiscence is integral in the decision to divert a downstream anastomosis. Surgeons were asked to quantitate this risk threshold. The median AL rate of $15 \%$ (interquartile range [IQR], 10 to 20 ) was felt to justify use of a TDI in low pelvic anastomoses and a median of $10 \%$ (IQR, 5 to 18$)$ for ileal pouches (Fig. 6). 


\section{Diatrizoate use}

Respondents very commonly use diatrizoate (Gastrografin) in clinical practice. Of surgeons, $91.1 \%$ use diatrizoate in patients with a small bowel obstruction or ileus, and $85.6 \%$ use it in the investigation of postoperative AL. Only 3.3\% of respondents would not use diatrizoate to confirm a defunctioned anastomosis has healed.

\section{DISCUSSION}

Surgeons make decisions based on their critical review and synthesis of contemporary literature, past experience, and the practice of their mentors and colleagues. The literature is the constant but there is wide variation in practice. How then do we rationalize this difference?

For instance, there is good evidence for the practice of employing rectal tubes [5] to reduce AL and the consequences of AL, but as many as $40 \%$ of surgeons in this study never use this modality. This is unexpected as the complications from rectal tubes are extremely low [17].

There is little information in the literature to elucidate patients' preference for a covering ileostomy and at what level of risk they would consider it worthwhile to 'divert' their low pelvic anastomosis. When surgeons were asked, at what risk of AL they considered a diverting ileostomy was in their patients' best interest, they considered above $15 \%$ as their cut-off. But few surgeons would quote an AL rate of $15 \%$ for all cases.

A 2015 systematic literature review found that the rate of AL varied between $1 \%$ and $19 \%$ depending on the anatomical site of the anastomosis. The authors reported an observed AL rate of 5\% to $19 \%$ for colorectal/coloanal and $4 \%$ to $7 \%$ for ileoanal pouches [18]. Early studies have urged caution with high AL rates in anastomoses involving the distal third of the rectum [19]. Variation in AL rates can, in part, be explained by varying definitions. The International Study Group of Rectal Cancer [20] defines AL as a defect of the intestinal wall at the anastomotic site leading to a communication between the intra- and extraluminal compartments.

Surgeons with a lower average AL rate would recognize that a mandatory policy of diverting ileostomy would be exposing a substantial number of lower-risk patients to a stoma that they do not need, with the additional risk of postoperative morbidity and requirement for a second operation. This would be reconcilable if it was not possible to determine which patients are at low risk. This issue has been studied extensively in the literature and there are numerous reviews, risk scoring tools, and online calculators available [21]. The REctal Anastomotic Leak score is a recently validated online tool. It is recognized that even low-risk patients do experience $\mathrm{AL}$, but they make up a small cohort compared to those predicted to be at higher risk.

There is significant geographical variation in practice. Of Western Australian surgeons, $71.4 \%$ have a policy of mandatory TDI, while only $14.3 \%$ of Queensland surgeons employ a mandatory
TDI in low pelvic colorectal anastomoses (Fig. 2). An even lower rate of mandatory TDI was observed when high-volume surgeons were considered (37.8\% vs. $27.2 \%, \mathrm{P}=0.28$ ) (Fig. 3 ), with no highvolume Queensland surgeons adopting a policy of mandatory TDI.

Surgeons, generally, are even more conservative with ileal pouches. Across the board, a high proportion of surgeons apply a mandatory policy of TDI. This is despite the fact that such a stoma is, by definition, high output and its construction difficult. These stomas will often lie flush with the skin and in some cases allow overflow so that the stoma does not even defunction the downstream intestine as intended. High-volume institutions, such as the Cleveland Clinic, report early AL rates for pouch surgery of $4.8 \%$ in 3,707 ileal pouch operations [22]. This is well below the threshold (10\%; Fig. 6B) most surveyed Australia and New Zealand surgeons would consider that a TDI is in their patients' best interest, yet overall $80.0 \%$ of these surgeons would defunction an ileal pouch with a TDI. The self-reported United Kingdom pouch audit revealed a TDI rate of $77.3 \%$ [23]; and in the Cleveland Clinic paper, they employed a TDI in $88.2 \%$ of their ileal pouches.

Again, in ileal pouch surgery, geographical variation in TDI policies was observed. While $100 \%$ of South Australian respondents have a policy of mandatory TDI, the figure is only $42.9 \%$ of Queens-land surgeons.

The risk of anastomotic dehiscence is integral in the decision to divert a downstream anastomosis. The lower the risk, the less likely a TDI will help the patient and thus they are relatively more likely to suffer the inherent complications of the procedure and its subsequent reversal.

In an attempt to understand why there are differences, surgeons were asked "at what percentage of AL did they consider a TDI was in their patients' best interest." These results were interesting. Fig. 6 shows clustering around a figure of $15 \%$ for low colorectal anastomoses and $10 \%$ for ileal pouches. At these figures, a significant majority of patients would have a stoma that they never needed. As a TDI does not reduce AL, the presumption is that significant harm will come to that small proportion who do leak. This would have to be considered to be over and above the morbidity of returning a previously undiverted patient to the operating theatre for peritoneal lavage and ileostomy.

Pelvic drain use is controversial. While a number of studies have shown no benefit, it is recognized that surgeons nonetheless prefer to leave a drain in some circumstances. In this survey, colorectal surgeons regularly use pelvic drains (Fig. 5). A further use for pelvic drains would be to allow sampling of the environment around the anastomosis for biomarkers. There is an ongoing search for either systemic or local biomarkers to allow early identification of AL. Pelvic drain fluid has been used to study biomarkers that may portent a clinical leak [24]. A local biomarker in the setting of IPAA is amylase, which can be measured simply and quantitatively in drain fluid. It has already been shown, in an early study from this institution, that amylase is present in high 
concentrations in enteric content from the rectal tube [25]. There is ongoing research to assess its utility in early diagnosis of AL.

Surgeons make choices for patients but there is little data to inform surgeons of the patients' preference for risk tolerance and involvement in the decision to defunction a low pelvic anastomosis.

There are a number of limitations to this study. As with any survey, there is a bias toward the respondents. There may have been surgeons with different policies who did not respond. The response rate of $31.6 \%$ is acceptable but lower than desired. Over a 9-year period of assessment, published CSSANZ surveys averaged a response rate of $52.5 \% \pm 18.3 \%$ (mean and standard deviation) [26]. The survey was only sent to members of CSSANZ, so the outcomes may not be generalizable across the field of general surgeons. Extending the survey was not felt necessary as most rectal cancer and virtually all ileal pouch operations would be performed by dedicated colorectal surgeons. Ricardo Hamilton et al. [26] have discussed the benefits of surveys as a study methodology and in particular their utility in setting the background and identifying areas of controversy to direct further research. This is the experience of Australia and New Zealand colorectal surgeons, and it is recognized that other countries will have differing preferences.

In conclusion, there remains considerable variation in colorectal surgical practice throughout Australia and New Zealand. While surgeons interrogate the same literature, there are presumably other factors that see translation into variations in clinical practice.

\section{CONFLICT OF INTEREST}

No potential conflict of interest relevant to this article was reported.

\section{ACKNOWLEDGMENTS}

The CSSANZ Research Support Committee is to be thanked for the deidentified distribution of the study questionnaire.

\section{REFERENCES}

1. Boccola MA, Buettner PG, Rozen WM, Siu SK, Stevenson AR, Stitz R, et al. Risk factors and outcomes for anastomotic leakage in colorectal surgery: a single-institution analysis of 1576 patients. World J Surg 2011;35:186-95.

2. Shogan BD, Carlisle EM, Alverdy JC, Umanskiy K. Do we really know why colorectal anastomoses leak? J Gastrointest Surg 2013; 17:1698-707.

3. Lovegrove RE, Constantinides VA, Heriot AG, Athanasiou T, Darzi A, Remzi FH, et al. A comparison of hand-sewn versus stapled ileal pouch anal anastomosis (IPAA) following proctocolectomy: a meta-analysis of 4183 patients. Ann Surg 2006;244:18-26.

4. Sagar PM, Pemberton JH. Intraoperative, postoperative and reoperative problems with ileoanal pouches. Br J Surg 2012;99:454-68.
5. Xiao L, Zhang WB, Jiang PC, Bu XF, Yan Q, Li H, et al. Can transanal tube placement after anterior resection for rectal carcinoma reduce anastomotic leakage rate? A single-institution prospective randomized study. World J Surg 2011;35:1367-77.

6. Wong NY, Eu KW. A defunctioning ileostomy does not prevent clinical anastomotic leak after a low anterior resection: a prospective, comparative study. Dis Colon Rectum 2005;48:2076-9.

7. Boyce SA, Harris C, Stevenson A, Lumley J, Clark D. Management of low colorectal anastomotic leakage in the laparoscopic era: more than a decade of experience. Dis Colon Rectum 2017;60:807-14.

8. den Dulk M, Smit M, Peeters KC, Kranenbarg EM, Rutten HJ, Wiggers T, et al. A multivariate analysis of limiting factors for stoma reversal in patients with rectal cancer entered into the total mesorectal excision (TME) trial: a retrospective study. Lancet Oncol 2007;8:297-303.

9. Fish DR, Mancuso CA, Garcia-Aguilar JE, Lee SW, Nash GM, Sonoda $\mathrm{T}$, et al. Readmission after ileostomy creation: retrospective review of a common and significant event. Ann Surg 2017;265: 379-87.

10. Jafari MD, Halabi WJ, Jafari F, Nguyen VQ, Stamos MJ, Carmichael JC, et al. Morbidity of diverting ileostomy for rectal cancer: analysis of the American College of Surgeons National Surgical Quality Improvement Program. Am Surg 2013;79:1034-9.

11. Fielding A, Woods R, Moosvi SR, Wharton RQ, Speakman CT, Kapur S, et al. Renal impairment after ileostomy formation: a frequent event with long-term consequences. Colorectal Dis 2020; 22:269-78.

12. Sahami S, Buskens CJ, Fadok TY, Tanis PJ, de Buck van Overstraeten A, Wolthuis AM, et al. Defunctioning ileostomy is not associated with reduced leakage in proctocolectomy and ileal pouch anastomosis surgeries for IBD. J Crohns Colitis 2016;10:779-85.

13. Sugerman HJ, Sugerman EL, Meador JG, Newsome HH Jr, Kellum JM Jr, DeMaria EJ. Ileal pouch anal anastomosis without ileal diversion. Ann Surg 2000;232:530-41.

14. Emile SH, Abd El-Hamed TM. Routine drainage of colorectal anastomoses: an evidence-based review of the current literature. Gastroenterol Res Pract 2017;2017:6253898.

15. Denost Q, Rouanet P, Faucheron JL, Panis Y, Meunier B, Cotte E, et al. To drain or not to drain infraperitoneal anastomosis after rectal excision for cancer: the GRECCAR 5 randomized trial. Ann Surg 2017;265:474-80.

16. Rondelli F, Bugiantella W, Vedovati MC, Balzarotti R, Avenia N, Mariani E, et al. To drain or not to drain extraperitoneal colorectal anastomosis? A systematic review and meta-analysis. Colorectal Dis 2014;16:035-42.

17. Shigeta K, Okabayashi K, Baba H, Hasegawa H, Tsuruta M, Yamafuji $\mathrm{K}$, et al. A meta-analysis of the use of a transanal drainage tube to prevent anastomotic leakage after anterior resection by double-stapling technique for rectal cancer. Surg Endosc 2016;30: 543-50.

18. McDermott FD, Heeney A, Kelly ME, Steele RJ, Carlson GL, Winter DC. Systematic review of preoperative, intraoperative and 
postoperative risk factors for colorectal anastomotic leaks. $\mathrm{Br}$ J Surg 2015;102:462-79.

19. Laxamana A, Solomon MJ, Cohen Z, Feinberg SM, Stern HS, McLeod RS. Long-term results of anterior resection using the double-stapling technique. Dis Colon Rectum 1995;38:1246-50.

20. Rahbari NN, Weitz J, Hohenberger W, Heald RJ, Moran B, Ulrich A, et al. Definition and grading of anastomotic leakage following anterior resection of the rectum: a proposal by the International Study Group of Rectal Cancer. Surgery 2010;147:339-51.

21. Arezzo A, Migliore M, Chiaro P, Arolfo S, Filippini C, Di Cuonzo $\mathrm{D}$, et al. The REAL (REctal Anastomotic Leak) score for prediction of anastomotic leak after rectal cancer surgery. Tech Coloproctol 2019;23:649-63.

22. Fazio VW, Kiran RP, Remzi FH, Coffey JC, Heneghan HM, Kirat HT, et al. Ileal pouch anal anastomosis: analysis of outcome and quality of life in 3707 patients. Ann Surg 2013;257:679-85.

23. Worley GHT, Fearnhead NS, Brown SR, Acheson AG, Lee MJ,
Faiz OD, et al. Review of current practice and outcomes following ileoanal pouch surgery: lessons learned from the Ileoanal Pouch Registry and the 2017 Ileoanal Pouch Report. Colorectal Dis 2018; 20:913-22.

24. Komen N, Slieker J, Willemsen P, Mannaerts G, Pattyn P, Karsten $\mathrm{T}$, et al. Acute phase proteins in drain fluid: a new screening tool for colorectal anastomotic leakage? The APPEAL study: analysis of parameters predictive for evident anastomotic leakage. Am J Surg 2014;208:317-23.

25. Clark DA, Cuda T, Riddell A, Radford-Smith G, Solomon M. Drain fluid amylase as a sensitive biomarker for the early detection of anastomotic leakage in ileal pouch surgery. Colorectal Dis 2019; 21:460-4.

26. Ricardo Hamilton AE, Lin AA, Young CJ. The benefits of colorectal surgery surveys in Australia and New Zealand. Ann Coloproctol 2020;36:102-11. 
Appendix 1. Rectal tubes, defunctioning stomas and drain use in low pelvic colorectal resections and ileal pouches

CSSANZ code:

1. Surgeon age:

2. Location of practice:

3. Years in consultant practice:

4. Number (approx.) rectal resections per year:

5. Number (approx.) ileal pouches per year:

\section{Ileostomy Preferences:}

The use of a covering loop ileostomy may not prevent an anastomotic leak but there is some evidence that it mitigates the consequences.

6. In patients who have not had pre-operative radiotherapy, do you use or plan to use a covering loop ileostomy for low pelvic colorectal anastomoses (ie. ULAR or low anterior resections) ?

All cases/mandatory $\quad \square$

Selectively $\square$

percentage (approx. proportion of cases)

Never

7. A loop ileostomy in ileal pouch surgery is more difficult to construct than after a colorectal anastomosis. Do you use or plan to use a covering loop ileostomy for ileal pouch procedures (IPAA) in:
All cases/mandatory
Selectively
Never
percentage (approx. proportion of cases)

\section{For low colorectal anastomoses:}

8. At what risk, or greater, of anastomotic leak in low pelvic colorectal anastomoses do you consider a defunctioning ileostomy is in the patient's best interest: $\%$;

\section{For lleal Pouches:}

9. At what risk, or greater, of anastomotic leak, in ileal pouches, do you consider a defunctioning ileostomy is in the patient's best interest: $\%$;

\section{Rectal tube usage:}

10. Rectal tube use in low pelvic anastomoses

$\begin{array}{cc}\text { All } & \square \\ \text { Selectively } & \square \\ \text { Never } & \square\end{array}$

If do use a rectal tube, how long is it left?

5 days

7 days

Other: days Comment: 
11. Rectal tube use in Ileal pouches:

All

Selectively

Never

$\square$

$\square$

$\square$

If do use a rectal tube, how long is it left?

5 days

7 days

Other: days Comment:

\section{Pelvic Drains:}

12. There remains controversy in the use of drains in pelvic surgery. Do you use or plan to use drains in: All/mandatory Selectively Never

If you do use a pelvic drain, how long is it left?

Minimum 5 days $\square$

Minimum 7 days $\square$

Until stops draining

Other days Comment:

\section{Gastrografin Usage:}

13. Do you use Gastrografin in ileus or SBO? Yes $\square \quad$ No $\square \quad$ Sometimes $\square$

14. Do you use rectal Gastrografin for diagnosing an anastomotic leak (in combination with any imaging modality)? Yes $\square$ No $\square$

15. Do you use Gastrografin (in combination with any imaging modality) to confirm a defunctioned anastomosis has healed?

Yes $\square$ No $\square$

Thank you for your time, these results will be presented at the Spring Meeting in Fremantle.

David Clark \& Bree Stephensen 